\title{
Triglyceride Metabolism
}

National Cancer Institute

\section{Source}

National Cancer Institute. Triglyceride Metabolism. NCI Thesaurus. Code C40657.

Triglyceride Metabolism involves anabolic and catabolic biochemical changes to triglycerides. 